\title{
REFLEXIONES RESPECTO A LA CONSTRUCCIÓN DE CONOCIMIENTOS EN EL ÁREA DE LA ENSEÑANZA DE LAS ARTES PLÁSTICAS
}

\author{
Julieta Castro Bonilla \\ Investigadora en el Instituto de Investigación \\ en Educación (INIE).
}

Recibido 21-IX-2004 • Aceptado 9-XI-2004

\begin{abstract}
Resumen: Las innovaciones que se producen día con día en los campos científico, tecnológico, ambiental, social, entre otros, afectan de manera significativa la educación y en consecuencia la labor que desempeñan los docentes. En este marco se le reconoce a la educación su función de orientar e impulsar politicas que respondan a las necesidades e intereses de quienes conforman el contexto social. El presente artículo pretende que quien imparte lecciones en el área de las Artes Plásticas comprenda que, como facilitador de los procesos de enseñanza y aprendizaje, debe fundamentar su práctica docente en referentes teóricos que les permitan a sus estudiantes trascender su análisis teórico y crítico, y llegar a propuestas concretas, realizables y transferibles a otros contextos. No debe nunca considerarse dueño absoluto de la verdad y del conocimiento. Debe eso sí tomar en consideración, de acuerdo con su formación y experiencia en esta área, referentes teóricos como el constructivismo, el holismo, la teoría de las inteligencias múltiples, la aplicación de la pedagogía, la psicología, la epistemología y la didáctica, que entrelazados con los contenidos propios del área de las Artes Plásticas, le permitan organizar un trabajo de aula reflexivo, que ofrezca la posibilidad de generar aprendizajes importantes. Por consiguiente, le corresponde al profesor de Artes Plásticas estudiar e investigar en forma rigurosa los fundamentos mencionados, así como practicar de manera constante técnicas artísticas variadas, pues le facilitan al estudiante el desarrollo de sus habilidades para aprender, para asimilar información eficiente y oportuna, para tomar decisiones, solucionar problemas e incrementar su creatividad.
\end{abstract}

Palabras clave: Constructivismo, holismo, teoría de las inteligencias múltiples, pedagogía, psicología, epistemología, didáctica, enseñanza de las Artes Plásticas.

\section{Introducción}

Con el propósito de brindar al sistema educativo costarricense un enfoque curricular novedoso, que respondiera a las necesidades e intereses de nuestra población estudiantil y ofreciera opciones para una educación de calidad, así como a la formación de ciudadanos capaces de transformar su realidad considerando los principios éticos para la convivencia, el Ministerio de Educación Pública establece a partir del año 1990 un enfoque curricular constructivista, que tiene como propósito revitalizar la práctica docente de los educadores mediante metodologías innovadoras, que permitan el logro de aprendizajes significativos de parte de los estudiantes. Molina S. (1999, p. 4) expone, “...se asume el constructivismo como un enfoque curricular, esto es, se adopta una posición teórica que da énfasis a los elementos y procesos curriculares, específicamente, a los procesos de enseñanza y aprendizaje". De acuerdo con lo anterior y acorde con los lineamientos propuestos por el mismo Ministerio, le corresponde al docente asumir este reto que, en la mayoría de los casos, se da de manera algo improvisada. Poco a poco este enfoque se lleva a la práctica mediante propuestas pedagógicas que permiten el fortalecimiento de los desarrollos cognitivo, socioafectivo y 


\begin{abstract}
The innovations produced day by day in the scientific, technological, environmental and social fields, among others, affect not only education but also teachers' performance. In this context, education plays an important role in encouraging policies that can respond to the needs and interests of those are involved in this social context. This article intends to help the fine arts teacher to understand that as facilitator of the teaching learning process, his or her training practice must be based on theoretical referents that allow his or her students to go beyond the theoretical-critical analysis and thus reach substantial and attainable proposals which could be transferred to other contexts. Moreover, this teacher does not have to consider him / herself the absolute owner of truth and knowledge. In order to generate important learning experiences for the students, s/he should consider interviewing theories such as constructivism, holism, multiple intelligences, pedagogy, psychology, epistemology and other didactic methods.

The fine arts teacher is responsible for studying and researching rigorously these theories, as well as practicing a variety of artistic techniques regularly. The theories will ease the development of students' learning abilities so that they assimilate information effectively, make decisions, solve problems and increase their creativity.
\end{abstract}

Key words: Constructivism, holism, multiple-intelligence theory, pedagogy, psychology, epistemology, methodology, teaching of fine arts. psicomotor en los estudiantes. No obstante lo anterior, a 14 años de permanecer el constructivismo como el enfoque curricular oficial, aún no se evidencian transformaciones pedagógicas significativas en el trabajo que desarrollan gran parte de nuestros docentes, pues se continúan llevando a la práctica metodologías en las que predomina una conducción de aprendizajes totalmente vertical, orientados por docentes que, en muchos casos, se consideran la única fuente de saber, situación que favorece con mayor énfasis el desarrollo del área cognitiva, dejando en desventaja la socioafectiva y la psicomotora.

El artículo que presento se dirige de manera especial a los profesores que imparten lecciones de Artes Plásticas, con el propósito de que reflexionen respecto a la práctica docente que organizan como parte de sus tareas cotidianas. Se espera que el texto se convierta en un insumo que les permita reflexionar y construir de manera científica y creativa procesos de enseñanza que faciliten aprendizajes desde una perspectiva integral.

El documento se refiere específicamente a la fundamentación teórica que origina el constructivismo, así como a otros conocimientos que, según Maya A., el docente debe conocer, pues le permiten organizar una práctica pedagógica efectiva; se refiere a la epistemología, pedagogía, psicología y a la didáctica, que entrelazados con los contenidos propios del área de las Artes Plásticas, dan vida y fortalecen el proceso didáctico. Se considera que el planteamiento metodológico que se expone le permite al docente de Artes Plásticas comprender que su principal misión es apoyar al estudiante en el desarrollo de una personalidad integral, para lo que es recomendable la organización de actividades bajo un plan de integración de conocimientos, habilidades y destrezas referidas al área artística. Lo anterior favorece en los alumnos la toma de decisiones, la solución de problemas propios del área de las artes plásticas, el fortalecimiento de sus 
formas de comunicación y la adquisición de un pensamiento más flexible y creativo.

\section{La educación holista}

Se inicia este apartado refiriéndose al concepto de educación holista que visualiza el pensamiento de manera integrada, no fraccionado; supone que el ser humano aprende integralmente, de los diversos escenarios de la vida cotidiana incluyendo su familia y el contexto escolar, que propician experiencias y nutren su historia personal. En estos escenarios se aprende de todo, valores, actitudes, creencias, concepciones y conocimientos, por lo tanto, la adquisición de esos aprendizajes se lleva a cabo de manera participativa y dinámica, ya que enfatiza la integración de las áreas cognitiva, socioafectiva y psicomotora, consideradas vitales para el desarrollo pleno de los estudiantes. Es importante indicar que el constructivismo parte de la "concepción de que la persona humana" es considerada una unidad que se manifiesta en tres dimensiones: la intelectual, la socioafectiva y la corporal, por lo que reconoce a la persona de manera integral, holística y totalizadora. El concepto de "persona humana abordado en la educación holista y en el constructivismo, son coincidentes al considerar que la concepción de naturaleza, de inteligencia, de aprendizaje, de conocimiento, de escuela, de comunidad, de alumno y de profesor, pueden ser concretados bajo el planteamiento pedagógico denominado constructivista, que implica precisamente un planteamiento curricular novedoso". Gallegos, R. (1999, p. 35).

También es fundamental incluir en este apartado el concepto de aprendizaje definido por Pérez. (1993, p. 81) como un "...proceso de transformación más que de acumulación de contenidos". En este sentido el estudiante es considerado un activo procesador de la información que asimila y el profesor es visualizado como un investigador del proceso dialéctico, a través del cual se transforman los pensamientos y las creencias del estudiante en experiencias concretas". Para que lo anterior se pueda llevar a la práctica es importante que el docente dedique parte de su trabajo de aula a investigar el estado actual del conocimiento y habilidades de sus estudiantes, incluyendo sus intereses y expectativas, ya que la información que asimilarán tendrá sentido en la medida en que pueda provocar cambios y transformaciones en la ya existente, lo que resultará aún más significativo.

La educación holista visualiza los tres aspectos centrales de todo proceso educativo: la inteligencia, el aprendizaje y el conocimiento; para estos la educación propone una concepción no fragmentada e integral de la naturaleza de la conciencia humana, lo que ayuda a comprender de manera global el proceso educativo del estudiante. Respecto a la inteligencia, Howard Gardner y un grupo de educadores, participan en un proyecto en el que desarrollan una nueva visión de inteligencia que denominaron "teoría de las inteligencias múltiples". Esta teoría reconoce muchas facetas distintas de la cognición, así como potenciales y estilos cognitivos en las personas. El resultado de esa investigación fue el reconocimiento de la existencia de siete tipos de inteligencias, concepto que se opone a la visión uniforme que se tenía de la inteligencia, pues se reconocían básicamente dos tipos: la lógica-matemática y la verbal, según indica Gallegos R. (1999).

Concluyo este apartado indicando que el profesor de Artes Plásticas debe ofrecer metodologías interactivas, mediante las cuales sus estudiantes se puedan expresar desde un planteamiento integral, o sea, estableciendo un equilibrio entre las áreas intelectual, socioafectiva y psicomotora, por lo que debe organizar espacios y momentos, dentro y fuera del aula, que den como resultado la vivencia de los objetivos propios del área de la artes plásticas, que precisamente enfatizan con claridad esas tres áreas. 


\section{La teoría de las inteligencias múltiples}

Como se indicara al inicio, si el docente lleva a la práctica el constructivismo como el enfoque curricular que propone el Ministerio de Educación Pública, considera la importancia de las percepciones, pensamientos y emociones del alumno en los intercambios que se producen durante la enseñanza y el aprendizaje. En otras palabras, el constructivismo indica que el estudiante debe construir conocimiento por sí mismo, y con la ayuda de otro (mediador), y que solo podrá aprender elementos que estén conectados a conocimientos, experiencias o conceptos previamente adquiridos por él. Lo que el alumno aprende no es una copia de lo que observa a su alrededor, sino el resultado de su propio pensamiento y razonamiento, así como de su mundo afectivo. En consecuencia, el docente debe permitir que el escolar investigue, que encuentre soluciones y haga sus propias conexiones para generar un significado interno que es único, porque ha sido construido por él y con la ayuda de otros. Por consiguiente, la función del docente es guiar los procesos de enseñanza mediante preguntas, guías, conduciendo e interactuando con sus estudiantes, no enseñando, como indica Martin,1997 citado por Klingler C. $(2000,8)$.

Para que el trabajo de aula se pueda concretar de una manera placentera y lúdica, se sugiere la aplicación de la "teoría de las inteligencias múltiples", ya que visualiza muchas facetas de la cognición, así como potenciales estilos cognitivos en las personas, lo que favorece un mejor aprendizaje. Los logros del trabajo investigativo coordinado por Howard Gardner, dieron como resultado el reconocimiento de la existencia de siete tipos de inteligencias. Según esta teoría, los seres humanos tenemos variadas formas de aprender, o sea, poseemos múltiples inteligencias que combinamos y aplicamos de diferentes maneras y de acuerdo con la organización de las actividades. Por consiguiente, el docente debe tener una actitud investigativa y creativa, de manera que sea capaz de organizar de forma equilibrada actividades que conlleven al fortalecimiento de las diversas capacidades de aprender en sus estudiantes. Si se lleva a la práctica la "teoría de las inteligencias múltiples" se evidencia que no existe un conjunto único de estrategias pedagógicas que funcione, pues según esta teoría, no existen estrategias metodológicas "únicas", de manera que algunas pueden funcionar muy bien con un estudiante o con un grupo, pero no tan bien con otro; por consiguiente, el docente como gestor de procedimientos metodológicos debe organizar actividades que involucren el desarrollo de diversas inteligencias, lo que le permitiría al estudiante asumir los fracasos académicos con mayor fortaleza, pues participaría en actividades que involucrarían varias inteligencias. Armstrong T. (1995).

Según lo expuesto, el ambiente de aula se debe convertir en un centro de investigación y de actividades que permitan el enriquecimiento individual y social, en donde el error académico y técnico se conviertan en motivos de reflexión, y la contextualización y vivencia de los objetivos propios de las Artes Plásticas consideren diversos canales de aprendizaje: el auditivo, el visual, el kinestésico y el táctil.

Como se indicara anteriormente, mientras la visión uniforme de la inteligencia reconocía básicamente dos tipos: la lógico-matemática y la verbal, la visión de H. Gardner es plural e incluye por primera vez, aspectos no cognitivos. El espectro de habilidades que poseemos los seres humanos es agrupado por este investigador en las siguientes siete categorías o inteligencias:

- Inteligencia lingüística: se considera la capacidad de emplear palabras eficazmente o bien en forma oral. (p.ej. cuentista, orador o político) o escrita (p.ej. poeta, dramaturgo, editor o reportero). Esta inteligencia comprende a la habilidad de 
manipular la sintaxis o estructura del lenguaje, la fonética o sonidos del leguaje, la semántica o significados del lenguaje, está basada en el razonamiento lógico, es vista como capacidad científica. Su referente biológico se encuentra en varias áreas del cerebro no verbal. Es el tipo de inteligencia de los test tradicionales.

- Inteligencia lógica y matemática: se refiere a la capacidad de emplear números y para razonar bien. Esta inteligencia abarca sensibilidad a las relaciones y patrones lógicos, enunciados y propuestas (si...entonces; causa $\mathrm{y}$ efecto), funciones $\mathrm{y}$ otras abstracciones afines. Los tipos de procesos utilizados en la aplicación de la inteligencia lógica y matemática incluyen: la agrupación por categorías, la clasificación, la interferencia, la generalización, el cálculo y la comprobación de hipótesis.

- Inteligencia espacial: habilidad de percibir acertadamente el mundo visual y espacial (cazador, explorador o guía) y para transformar esas percepciones (decorador de interiores, arquitecto, artista o inventor). Esta inteligencia implica sensibilidad al color, línea, forma, figura, espacio y la relación que existe entre estos elementos. Incluye la capacidad para visualizar, para representar gráficamente las ideas visuales o espaciales y para orientarse uno mismo correctamente en una matriz espacial.

- Inteligencia física y cinestética: experiencia utilizando el cuerpo propio para expresar ideas y sentimientos (actor, mimo, atleta o bailarina) y facilidad de emplear las manos para producir o transformar cosas (p.ej. artesano, escultor, mecánico, cirujano). Esta inteligencia comprende habilidades físicas específicas como la coordinación, el equilibrio, la destreza, la fuerza, la flexibilidad y la velocidad, así como también habilidades táctiles y hápticas.

- Inteligencia musical: capacidad para percibir, distinguir y expresar notas musicales. Esta inteligencia comprende sensibilidad al ritmo, compás o melodía y al timbre o tonalidad de una pieza musical. La música puede comprenderse en forma figurada (global intuitiva), o de manera formal (analítica, técnica) o ambas.

- Inteligencia interpersonal: es considerada como la habilidad de percibir y distinguir los estados de ánimo, intenciones, motivos y sentimientos de otras personas. También puede abarcar a las expresiones faciales, a la voz y a los gestos: la capacidad para discriminar entre muchas clases diferentes de signos interpersonales y la habilidad de responder eficazmente a esas señales de alguna manera programática (influir en algún grupo de gente para que sigan alguna línea de acción).

- Inteligencia intrapersonal: conocimiento propio y la habilidad de actuar adaptadamente sobre la base de ese conocimiento. Esta inteligencia comprende tener una imagen acertada de sí mismo (de las fortalezas y limitaciones) reconocimiento de los estados de ánimo, intenciones, motivaciones, temperamentos y deseos; y la capacidad de autodisciplina, autocomprensión y amor propio. Amstrong T. (1995, p. 2, 3).

Aunque el presente artículo se dirige especialmente a los docentes de Artes Plásticas y éstos enfatizan el desarrollo de la inteligencia espacial, se incluye la descripción de las siete inteligencias por considerar que poseemos más de una inteligencia, y 
como gestor de propuestas pedagógicas y artísticas, le corresponde al docente organizar actividades de enseñanza que favorezcan diversas formas de aprender.

\section{Respecto al concepto de enseñanza de las Artes Plásticas}

Se inicia este apartado con una referencia general al concepto de enseñanza, visualizado como un proceso de interacción que se produce entre un adulto, con mayor formación académica, artística y experiencia, y un joven que desea adquirirlas. Se parte de la definición anterior para enfatizar en la enseñanza de las Artes Plásticas, considerando que en esta área el docente debe estimular a los alumnos para que se identifiquen con sus propias creaciones y experiencias. Asimismo, debe animarlos para que expresen de manera concreta y con libertad sus conocimientos, sus sentimientos, sus emociones y su propia sensibilidad estética. En el proceso educativo lo que importa es el aprendizaje del estudiante y el esfuerzo del joven por lograr su propia respuesta. De acuerdo con lo expuesto, quien imparte lecciones en esta área expresiva debe poseer una formación pedagógica y artística idónea, que sea capaz de ofrecer a sus estudiantes procedimientos didácticos cargados de creatividad, con el propósito de que participen en la construcción de conocimientos referidos al área artística, así como a la adquisición de destrezas y habilidades que impliquen el desarrollo de su sensibilidad. Por ello, es fundamental la aplicación de técnicas y materiales apropiados para la creación de sus productos artísticos. Es importante indicar que mediante la producción de sus propios trabajos de arte (pinturas, esculturas, grabados, dibujos, impresiones, otras), los estudiantes adquieren un mayor acercamiento a las obras de arte de artistas reconocidos o no. De esta manera podrán explorar, experimentar y expresar con facilidad sus ideas, sentimientos y emociones, pues su experiencia artística les permite un mayor acercamiento a la cultura, en donde el estudiante podrá combinar sus experiencias y construir nuevas realidades que correspondan a sus necesidades y curiosidades. Por lo tanto, es conveniente que este crezca en un ambiente rico en motivaciones, oportunidades y estímulos en todas direcciones, como afirman Pacheco J. y Pacheco M. (1998,11). En consecuencia, si los estudiantes participan de manera activa en sus propias experiencias artísticas, se podrán "autoidentificar" no solo con sus creaciones, sino con las que realizan sus compañeros, de manera que sean capaces de decir con orgullo y satisfacción "esto es mío", lo que les permitirá plantearse nuevos propósitos, retos e intereses, así como ser responsables de sus formas de expresión y de los procedimientos empleados. Por consiguiente, todo docente de arte debe ser capaz de crear un ambiente en el cual los conocimientos, los pensamientos y los sentimientos, se puedan expresar con libertad, mediante diversos materiales y formas; lo mismo que puedan ser expuestos y tratados con sensibilidad y respeto. Le corresponde al educador la tarea de planificar actividades que le permitan al estudiante el logro de los objetivos de expresión artística, por lo que debe tomar en cuenta su capacidad intelectual, su forma de aprender, sus intereses, dificultades, necesidades personales, edad, así como el contexto escolar y el nivel socioeconómico.

Torrent, $\mathrm{M}^{\mathrm{a}}$. E. y Chehuaras M. (2000, 26), consideran que se debe enseñar arte porque:

- $\quad$ es propio de nuestra cultura y forma parte de nuestra historia.

- $\quad$ es un lenguaje visual, que desarrolla la capacidad expresiva y sensitiva.

- $\quad$ porque su percepción ayuda al desarrollo del conocimiento.

- tiene unos valores propios, diferentes del lenguaje oral, escrito, de la lógica y del conocimiento científico. 
proporciona un conocimiento del mundo.

- facilita el desarrollo creativo del alumnado.

De acuerdo con lo expuesto, el docente debe organizar y planificar su práctica pedagógica, recordando que en todo proceso de enseñanza y aprendizaje se evidencian cuatro elementos que merecen especial atención: el estudiante, el docente, el conocimiento y el desarrollo de destrezas. La enseñanza como práctica social y por formar parte de la educación, se debe organizar tomando en cuenta, no solo los elementos mencionados, sino la planificación de unidades didácticas en las que la organización de objetivos, contenidos, actividades, recursos y evaluación, de manera coherente y secuencial, son fundamentales para el trabajo de aula. Los temas que se refieren a la expresión plástica, así como las destrezas y habilidades debidamente entrelazadas y organizadas de manera prospectiva, flexible y creativa, en contextos escolares óptimos, dan como resultado el logro de objetivos, y por lo tanto de aprendizajes. Para concluir el presente apartado, se mencionan los objetivos de la enseñanza de la Artes Plásticas, considerados el punto de partida para que el docente planifique y organice las actividades de tal manera que sus estudiantes los puedan alcanzar de manera vivencial.

\section{Objetivos generales de las Artes Plásticas:}

- Proporcionar actividades de taller (teóricopráctico) con temas incentivadores, utilizando los elementos del leguaje plástico y los elementos estéticos.

- Impulsar experiencias artístico-plásticas que contribuyan al desarrollo sensorial, motriz y afectivo (goce estético, apreciación, conocimiento teórico y producción artística).

- Promover el arte y los aportes artísticos nacionales y extranjeros en el tiempo y el espacio para el enriquecimiento cultural y el estímulo a la creatividad.
- $\quad$ Promover la valoración de las artes plásticas desde una visión humanista y científica.

- $\quad$ Promover actividades artísticas que favorezcan la realidad ecológica del medio y su relación con el mundo.

- Promover la participación de actividades artísticas en al cuales se manifiesten los valores estéticos, morales, éticos e intelectuales de la sociedad costarricense como parte de un país democrático.

- Preservar las costumbres, las tradiciones, el folklore y los valores culturales, mediante la realización de experiencias estéticas.

(Ministerio de Educación Pública. 2001).

\section{El enfoque constructivista, sus fundamentos}

Al referirme al constructivismo como el enfoque curricular que debe fundamentar el trabajo de aula de nuestros educadores, deseo compartir con los lectores una cita que Maya A. (2000 p. 53.) expone referente a la gestión que tiene el docente como facilitador de los procesos didácticos, y que debe conocer y llevar a la práctica de manera sistemática:

“...la práctica docente como el eje operativo de los procesos de enseñanza y aprendizaje o del proceso docente educativo, no se refiere exclusivamente a la aplicación de técnicas, tareas o actividades que se llevan a la práctica de una manera empírica o aislada, por repetición de experiencias intuitivas, sin el debido sustento comprensivo y explicativo de una teoría o conceptualización, validada científicamente, por ello, es necesario comprender que ninguna práctica debe darse sin una teoría que la acompañe o sin una base sobre la cual se construye, toda práctica docente debe poseer un planteamiento teórico que la sustente".

Para que la cita anterior pueda ser concretado por los docentes de Artes Plásticas, se hace referencia a algunos aportes teóricos que lo fundamentan, de la misma manera que fundamentan el constructivismo y lo conceptúan como un 
enfoque curricular, pues hace énfasis en los procesos de enseñanza y aprendizaje.

Los aportes de autores que a continuación se indican, así como otros conceptos analizados anteriormente (educación holista, la teoría de las inteligencias múltiples, y otros que se desee incorporar), permiten al profesor organizar su práctica docente desde un planteamiento científico, (Maya A.).

De acuerdo con Maat H. (1993), el enfoque constructivista no dispone de un marco teórico unificado que permita dar cuenta de los múltiples y complejos aspectos implicados en el trabajo de aula y en otras actividades que se organizan dentro y fuera del ámbito escolar, por lo que se debe acudir a contribuciones de educadores e investigadores para que sustenten su fundamentación desde una perspectiva interdisciplinaria.

Con el propósito de establecer un marco de referencia que permita comprender de mejor manera sus implicaciones en el trabajo de aula, se hace una breve referencia a algunos de los aportes de Jean Piaget, Lev. S. Vigotsky, Jerome Bruner y David Ausubel, considerados estudiosos y sobresalientes dentro de este enfoque.

a. De acuerdo con la Teoría psico-genética de Jean Piaget, conocer implica la participación de tres elementos: el sujeto que conoce, el objeto por conocer y el proceso de conocimiento que se origina de la relación de los dos primeros. Por lo tanto, el conocimiento es producto de la interacción social y de la cultura: todo lo que le es enseñado al niño, se le impide descubrirlo. De lo anterior, se interpreta que el docente no debe transmitir conocimientos, sino estimular el proceso de descubrimiento. ¿Cómo se construye el conocimiento de acuerdo con este autor? Sus aportaciones se resumen de la siguiente manera:

- Las experiencias educativas formales sobre el crecimiento personal del niño están fuertemente condicionadas por su nivel de desarrollo operativo.

- $\quad$ El individuo construye su propio conocimiento sobre el entorno físico y en relación con el entorno social.
- El conocimiento es el producto de la interacción social y cultural.

- La inteligencia es activa.

- $\quad$ El pensamiento se deriva de la acción del niño y no de su lenguaje.

- $\quad$ El pensamiento es diferente de acuerdo con la edad.

- El ser humano busca permanentemente el equilibrio.

b. La Teoría de la Significancia de Jerome Bruner, afirma que el conocimiento es construido de la siguiente manera:

- $\quad$ El niño aprende mediante el descubrimiento guiado.

- $\quad$ Por su propia actividad percibe, razona y construye.

- El niño no es un simple reactor al ambiente.

- El conocimiento es un proceso, no un producto.

- La educación es una forma de diálogo, en el que el niño aprende a construir conceptualmente el mundo con la ayuda y guía del adulto.

c. La Teoría Socio-genética de Vygotsky, señala los siguientes aspectos básicos:

- El lenguaje cumple una doble función: de comunicación y de conocimiento.

- La función del docente es fomentar el diálogo entre los niños, actuando como mediador y como potenciador en los procesos de enseñanza y aprendizaje.

- El aprendizaje debe considerarse como una actividad social.

- Se deberá tomar en cuenta el concepto denominado: "zona de desarrollo próximo", distancia entre el nivel real de desarrollo y el nivel de desarrollo potencial. Esto quiere decir que el individuo posee dos niveles de desarrollo del conocimiento. Uno es el real, el actual, que indica el logro que el alumno ha obtenido por sí mismo; el otro es el potencial que muestra lo que éste es capaz de aprender y hacer con ayuda de 
los demás, o sea, con la colaboración de quienes lo rodean.

d. David Ausubel citado por Dávila S., indica que las propuestas de Bruner sobre el Aprendizaje por Descubrimiento cobraban adeptos en forma acelerada, las experiencias se orientaban a que los niños en las escuelas construyeran su conocimiento a través del descubrimiento de contenidos, de esta manera se privilegiaron los descubrimientos dentro del aula. Ausubel reconoce las bondades del aprendizaje por descubrimiento, solo que el proceso requiere más tiempo. Algunas de las características del Aprendizaje Significativo son:

- Los nuevos conocimientos se incorporan en forma sustantiva en la estructura cognitiva del alumno.

- Esto se logra gracias a un esfuerzo deliberado del alumno por relacionar los nuevos conocimientos con sus conocimientos previos.

- Todo lo anterior es producto de una implicación afectiva del alumno, es decir, el alumno quiere aprender aquello que se le presenta porque lo considera valioso.

De acuerdo con la teoría de Ausubel, para lograr aprendizajes significativos es necesario que se cumplan las siguientes condiciones:

1. Significatividad lógica del material. Esto es que el material presentado tenga una estructura interna organizada, que sea susceptible de dar lugar a la construcción de significados (Coll, citado por Dávila S.). Los conceptos que el profesor presenta deben seguir una secuencia lógica y ordenada.

2. Significatividad psicológica del material. Esto se refiere a la posibilidad de que el alumno conecte el conocimiento presentado con los conocimientos previos, ya incluidos en su estructura cognitiva.

3. Actitud favorable del alumno. Que el alumno quiera aprender no basta para que se dé el aprendizaje significativo, pues también es necesario que pueda aprender. Para que el aprendizaje se pueda llevar a cabo es necesario que el alumno se encuentre dispuesto emocionalmente y actitudinalmente, en el que el maestro sólo puede influir a través de la motivación.
De acuerdo con lo expuesto, para que el docente organice de una manera novedosa su trabajo de aula, tiene la responsabilidad de conocer, reflexionar e indagar sobre los sustentos teóricos mencionados, ya que éstos deben tener presencia en los diferentes momentos y espacios que se organizan dentro y fuera del ámbito escolar.

Para que la práctica docente se defina de calidad no puede generarse de una manera empírica o producto de la rutina y de la casualidad, sino posterior al estudio y reflexión sistemática de teorías que sustenten su práctica docente, en la que debe privar una actitud razonada, que le permita asumir acciones que contribuyan a la vivencia de los objetivos de las Artes Plásticas, considerados el punto de partida para la construcción de las áreas cognitiva, socioafectiva y psicomotora, pilares que sustentan la educación holista.

Maya A. (2000), considera que la calidad de práctica docente o trabajo de aula, también se encuentra supeditada a los conocimientos que el docente posea sobre la epistemología, la pedagogía, la psicología, la didáctica, así como a las disciplinas específicas, pues intervienen directamente en su práctica docente. Para el presente artículo, la disciplina se refiere a los contenidos o temas que surgen a partir de los objetivos de las Artes Plásticas.

Es importante mencionar que el "enfoque curricular" denominado constructivismo, ha producido incertidumbre en algunos de nuestros educadores, pues al tener que planificar y organizar metodologías innovadoras, sustentadas en teorías, quizás desconocidas para muchos de ellos, han tenido que romper con prácticas pedagógicas tradicionales y difíciles de erradicar.

A continuación, se hace referencia a disciplinas que fundamentan y dan vida a las prácticas pedagógicas que el docente planifica para el logro de aprendizajes significativos de parte de los estudiantes.

- La epistemología o teoría del conocimiento es una disciplina derivada 
de la filosofía que estudia el "cómo es posible el conocimiento, cómo y cuándo podemos considerar nuestros conocimientos como verdaderos", en donde es fundamental establecer las relaciones que se pueden dar entre el sujeto que conoce y el objeto de conocimiento. El conocimiento epistemológico en el docente le permite no ser mecanicista, ni reproductor de los conocimientos, al contrario, le permite innovar constantemente su práctica, ya que lo hace reflexionar en cuanto a: ¿cómo construyen los estudiantes los conocimientos?, ¿cómo aprenden? y por lo tanto, ¿cómo se enseña?, ¿cómo se puede facilitar la enseñanza?

- La pedagogía según Ordoñes J. (1999), es la permanente sistematización interdisciplinaria de la experiencia que se tiene en la práctica educativa. Esa sistematización le permite al docente conceptuar o crear las condiciones teóricas que mejoran su trabajo de aula, dar significado y respuesta científica a su propia práctica docente, ya que le facilita construirla, deconstruirla y reconstruirla, con el propósito de adecuarla, adaptarla y responder a las necesidades de sus estudiantes, a partir de su bagaje intelectual, su experiencia, su edad y su contexto familiar y escolar.

A continuación se indican algunas de las bases pedagógicas que de acuerdo con el constructivismo pueden ser aplicadas en el contexto de aula:

- Se erradica la concepción de que el docente es el dueño absoluto de la verdad dentro del aula, así como el único sujeto activo, ya que se considera el desarrollo de la inteligencia y del aprendizaje como un proceso dialéctico en el que participan de manera activa tanto los alumnos como el docente.
- Como parte del planteamiento curricular, el educador debe asumir una actitud protagónica, puesto que sobre él recae la tarea de organizar su labor mediante una selección y adecuación de métodos que permitan construir conocimientos y habilidades por parte de sus estudiantes.

- Con gran responsabilidad, además de atender las necesidades de sus estudiantes, el profesor tiene la obligación de hacer ajustes y adecuaciones al planteamiento curricular emanado del Ministerio de Educación Pública, para lo que debe tomar en cuenta la edad, intereses y condición económica de sus estudiantes.

- La psicología le brinda al docente las herramientas necesarias para conocer a sus alumnos, así como explicar su comportamiento y comprender sus diferencias, sus actitudes y sus capacidades para aprender, asimilar, entender y comprender mejor los contenidos. Mediante el conocimiento de esta área, el docente podrá ayudar a solucionar problemas relativos a la disciplina escolar y permitir que el proceso didáctico se pueda producir en ambientes que motiven procesos de socialización, de respeto y de cooperación, que faciliten la formación de personalidades autónomas.

- La didáctica se refiere a la metodología como una área de la filosofía que trata de los métodos, los sistemas y las reglas para descubrir y construir aprendizajes. La didáctica, vista desde una perspectiva constructivista, le permite al docente la posibilidad de organizar actividades colectivas e individuales que propicien, vivencien y den significado a las experiencias que adquieren 
sus estudiantes. Maya A. (1999). La didáctica se nutre de las fuentes que sustentan la teoría constructivista, a saber: la Teoría psico-genética de Jean Piaget, la Teoría de la significancia de Jerome Bruner, la Teoría socio-genética de Vygotsky, y la Teoría cognitiva del aprendizaje de David Ausubel, ya que la aplicación de estas genera procesos de crecimiento personal, social, cognitivo, afectivo y psicomotor, lo que favorece la formación de personalidades con una visión holista.

La Teoría del aprendizaje que propone Ausubel, se centra en el aprendizaje que se produce en un contexto educativo en el que predominan los procesos de enseñanza y aprendizaje a partir de la práctica cotidiana, por lo que el docente debe establecer una relación constante entre los conocimientos que el estudiante ya posee o "previos" y los "nuevos o por aprender". En otras palabras, la materia o la práctica artística que se está conociendo debe relacionarse con otras experiencias, hechos, acontecimientos $\mathrm{u}$ objetos que la persona posee o conoce.

En el "aprendizaje significativo" la incentivación es considerada un factor esencial, ya que su incorporación a la estructura cognitiva de quien aprende es fundamental, y es precisamente al docente a quien le corresponde la responsabilidad de organizar ambientes que hagan posible la búsqueda de aprendizajes que tengan sentido y puedan surgir del propio estudiante, de su "yo" interno, de su motivación, ya que le permitirá aprender desde su propia estructura cognitiva, relacionando y estableciendo conexiones con experiencias que surgen de su ambiente familiar y social, lo que le proporcionará un verdadero aprendizaje. Como se indicara anteriormente, el docente debe convertirse en un constante cuestionador de su propia experiencia pedagógica, ya que plantear el acceso al conocimiento y a experiencias de arte de manera activa, participativa y creativa no es una tarea fácil.

Según lo expuesto, el docente debe interesarse por identificar en sus estudiantes cuál es la mejor forma de conocer y de expresar emociones y destrezas; en otras palabras, cuál es la mejor manera de acercarse a su propio conocimiento y a su propia creatividad, pues en el constructivismo, el aprendizaje se logra actuando, por lo que se deben planear y organizar de manera activa y creativa las actividades de enseñanza.

El término "planeamiento" se refiere a la organización sistemática, secuencial, coherente y prospectiva de sus componentes: objetivos generales y específicos, situaciones o actividades de iniciación, de desarrollo, de cierre, y finalmente los criterios de evaluación; cada uno de éstos debe fomentar la motivación constante, así como cultivar y despertar el interés y la atención de parte de los alumnos durante el transcurso de las actividades. En el planeamiento pueden intervenir los estudiantes en asocio con el profesor, por lo que "...es indispensable tener presente que la estructura cognitiva del alumno tiene una serie de antecedentes y conocimientos previos, un vocabulario y un marco de referencia personal, lo que es además un reflejo de su madurez intelectual". Díaz B. y Hernández R. (1998, p. 21).

De acuerdo con lo expuesto, el enfoque constructivista es en esencia dinámico y dialéctico, parte de la premisa de que no existen verdades absolutas, el método no está dado, se construye en forma activa y creativa con la experiencia del docente y tomando en cuenta la edad y las características psicológicas, cognitivas, afectivas y psicomotoras de sus estudiantes.

Tomando en cuenta mi experiencia como investigadora en I y II ciclos de la Educación General Básica, y como supervisora en III y IV ciclos de la Educación Diversificada, el "enfoque curricular" al que me he referido, aún no se encuentra totalmente arraigado en nuestras aulas, ya que su correcta aplicación, implica por 
parte del docente, suficiente tiempo y períodos extracurriculares para investigar, leer, reflexionar y proponer actividades placenteras, que involucren el contexto del alumno y de la institución, y no siempre nuestros educadores poseen actitudes que le inviten a la constante reflexión e innovación. Otras veces, las mismas políticas y directrices emanadas por la Institución o por las Direcciones Regionales impiden (por diversos motivos) cambios cualitativos en la labor que los docentes organizan. A los aspectos mencionados, se agrega que las cualidades personales del docente son esenciales: creatividad, emoción, paciencia, afecto, entusiasmo, y también las ocurrencias, que deben permanecer de manera constante durante el desarrollo de sus lecciones. Cabe destacar que muchas veces una o varias de estas cualidades se encuentran ausentes. Para concluir el presente apartado, el docente debe organizar su práctica pedagógica considerando como punto de partida, el enfoque curricular mencionado, las disciplinas que colaboran en la fundamentación de los procesos educativos, los conocimientos que posea sobre el área de las artes plásticas, incluyendo su experiencia, ingredientes fundamentales para que sus estudiantes puedan "vivir" de manera placentera los objetivos propios de las Artes Plásticas, considerados el punto de partida para desarrollar un trabajo de aula con carácter de eficiencia.

\section{El docente y su trabajo de aula}

Los planteamientos que se presentan no pretenden hacer "más compleja de lo que es" la tarea del docente, sin embargo, no debe perderse de vista que su principal función es apoyar al estudiante en el desarrollo de sus conocimientos, habilidades y destrezas, por lo que es importante que posea un gran dominio, no solo de los contenidos propios de esta área expresiva, sino de otros conocimientos que le permitan hacer de su "trabajo de aula, un taller de arte", en el que exista la investigación constante y la vivencia de contenidos teóricos y prácticos. Por consiguiente, quien imparte lecciones de Artes Plásticas debe planificar su labor permitiéndole al alumno que tome sus propias decisiones con respecto a su trabajo y a la solución de sus propios problemas. La programación de actividades debe incrementar la creatividad y el desarrollo del pensamiento divergente, sin olvidar los retos que debe enfrentar como parte de su cotidianidad, por lo que es conveniente presentar la información mediante variados recursos audiovisuales como fotografías, películas, dibujos y símbolos gráficos entre otros. Como se indicara anteriormente, la inteligencia espacial implica de parte de los estudiantes una sensibilidad especial hacia los colores, las líneas, las formas, los espacios, así como las relaciones que se producen entre esos elementos. Por lo tanto, conviene que el docente lleve a la práctica actividades que impliquen la concreción de conocimientos, imágenes mentales interiores y exteriores, ideas y sentimientos, mediante diversos materiales, sin importar el producto final sino el proceso que motivó ese producto. Es importante recordar la guía y la preocupación constante de parte del docente por enriquecer las posibilidades del trabajo artístico del estudiante, ya que se le debe dar la oportunidad de valorar su propia creación.

Klingler C. y Vadillo G. (2000. p. 180) expresan que no existe una teoría exclusiva $\mathrm{y}$ sustentable, y que no hay fórmulas universales para atender las diversas situaciones de aula; cada una debe tratarse como una experiencia única, que requiere soluciones específicas dependiendo del contexto, de los niveles de desarrollo de los estudiantes, de los temas por tratar y de los objetivos curriculares preparados con suficiente antelación por el docente. Si el profesor conoce el planteamiento pedagógico constructivista, si posee una visión holista de la educación y aplica la teoría de inteligencias múltiples, es capaz de darle soluciones viables a las 
situaciones difíciles que pueda encontrar en su contexto de aula.

En la educación holista el aprendizaje no es una función propia del conocimiento, éste también se lleva a cabo mediante procesos de tipo social, físico, emocional, cognitivo, estético y espiritual, por lo que le corresponde al docente ofrecer diversos estilos de enseñanza para que todos los estudiantes puedan aprender de la mejor manera posible.

Es importante recordar que el proceso de construcción de conocimientos se produce desde la experiencia del estudiante con él mismo y con los demás, es en la colectividad donde el proceso de individualización se lleva a cabo, es en el contexto social y cultural donde se origina el verdadero significado de la experiencia humana. Es mediante la cultura y el contacto con otros que se validan y privilegian determinadas formas de proceder. La aplicación de la teoría de las inteligencias múltiples permite la construcción del principio de la realidad del individuo, el desarrollo del pensamiento creativo, por lo que el contexto socio cultural no se puede obviar, si se pretende que los estudiantes construyan sus conocimientos le conviene al docente relacionar su área de estudio con otros contenidos de la misma materia y si es posible con otras disciplinas, con el propósito de que la construcción de conocimientos se pueda llevar a cabo de manera activa, dinámica y reflexiva.

\section{Conclusiones}

Los planteamientos que se presentan y que motivan el artículo se generan desde cuatro fuentes: la de investigadora en el área de la expresión artística, la de profesora de Artes Plásticas en instituciones de educación secundaria, desde mi experiencia como docente en la Escuela de Formación Docente de la Universidad de Costa Rica (en los cursos Introducción a la Pedagogía, Fundamentos de Didáctica, Metodología y Experiencia Docente con énfasis en Artes Plásticas). En el curso de Experiencia Docente los estudiantes deben llevar a la práctica los conocimientos teóricos y prácticos adquiridos durante el transcurso de su carrera, mediante una práctica supervisada semestral, la cual se convierte en un verdadero centro de experiencias, tanto para quien supervisa como para el estudiante universitario supervisado. Y, finalmente la literatura leída me ha permitido reflexionar y hacer los aportes que se presentan. Se espera que los mismos sean de beneficio para los docentes, especialmente a quienes se inscriben en el plan de estudios correspondiente al Bachillerato en la Enseñanza de las Artes Plásticas.

De acuerdo con los planteamientos expuestos conviene que el docente de Artes Plásticas comprenda que su trabajo de aula, tanto teórico como práctico, se debe fundamentar sobre teorías validadas científicamente, que eviten el empirismo y la repetición intuitiva de experiencias, sin el debido sustento explicativo de su trabajo.

Al conocer los objetivos de las Artes Plásticas le corresponde al docente organizar actividades teóricas y prácticas con temas incentivadores, utilizando los elementos del leguaje plástico, así como los elementos estéticos que contribuyan a su desarrollo sensorial, motriz y afectivo (goce estético, apreciación, conocimiento teórico y producción artística), por lo que le conviene la aplicación de la teoría de las inteligencias múltiples.

Por lo tanto, si el docente posee una formación idónea en el campo de las Artes Plásticas, si conoce y aplica el enfoque curricular constructivista, así como otras disciplinas entre las que se citan la epistemología, la pedagogía, la psicología y la didáctica, que le permiten comprender, concretar y hacer suyos los procesos educativos que se viven en el aula; si posee una visión holística de la educación, y considera como parte de su trabajo de aula la teoría de inteligencias múltiples, probablemente dará soluciones viables a los problemas y situaciones difíciles que pueda encontrar en su aula. El 
estudio, reflexión y aplicación de las teorías mencionadas le deben permitir al educador comprender que no existe un conjunto de estrategias ni técnicas artísticas que funcionen de manera excelente para la totalidad de los estudiantes. El aprendizaje es un proceso muy complejo, por lo que no existe un único método de enseñanza, la capacidad de preguntar, de hallar respuestas, de descubrir formas, de desechar para volver a hacer y encontrar nuevas soluciones nutren ese proceso. Probablemente una de las habilidades básicas que se deben enseñar en nuestros centros educativos sea la capacidad de descubrir y de buscar respuestas, en lugar de esperar pacientemente las contestaciones e indicaciones del docente. Según Lowenfeld V. y Lambert W., cada individuo posee un potencial que es mayor de lo que uno se imagina. La expresión artística brinda a cada estudiante la posibilidad de verse a sí mismo como un ser aceptable, que busca nuevos retos y logra tener confianza en sus propios medios de expresión.

\section{Referencias bibliográficas}

Armstrong, Thomas. "Inteligencias Múltiples en el salón de clases". Asociación para la supervisión y desarrollo de programas de estudio: Alexandría, Virginia USA. 1995.

Dávila E. Sergio. "El aprendizaje significativo", en Revista digital de Educación y Nuevas Tecnologías. N 9-julio 2000.

Díaz, B. Frida y Hernández, R. Gerardo. Estrategias Docentes para un Aprendizaje Significativo: Una interpretación constructivista. Mc GRAW-HILL. Companies Inc. México D.F. 1998.

Gallegos, N. Ramón. Educación Holista. Pedagogía del amor universal. Editorial Pax México D.F. 1999.

López, V. Carlos. "Constructivismo y su aplicación en el proceso de enseñanza-aprendizaje", en Revista Umbral: Educación y Constructivismo, Colegio de Licenciados y Profesores en Letras, Filosofía, Ciencias y Letras. II Semestre 1999. San José, Costa Rica.

Lowenfeld V. y Lambert W. Desarrollo de la Capacidad Creadora. Editorial Kapeluz. Buenos Aires, 1972.

Maat, Hendrien. Elementos de enfoque socio-constructivista en los aprendizajes. Ministerio de Educación Pública, Centro Nacional de Didáctica. Proyecto UNESCO/Países Bajos. San José, Costa Rica, 1993.

Maya, B. Arnobio y otros: "La Práctica Pedagógica Innovadora en el Aula y en la Escuela y su Sistematización”. UNESCO, Ministerio de Educación Pública. SIMED. San José, Costa Rica, 2000.

Molina, B. Zayda. "Planificación, Diseño y Desarrollo Curricular", en Revista Umbral: Educación y Constructivismo. Colegio de Licenciados y Profesores en Letras, Filosofía, Ciencias y Letras. II Semestre 1999. San José, Costa Rica.

Ordoñez, P. Jacinto. Introducción a la Pedagogía. Editorial Universidad Estatal a Distancia. San José Costa Rica. 1999.

Pacheco Julia y Pacheco Mary Luz. Construyendo imaginarios, Talleres creativos. Cooperativa Editorial Magisterio. Bogotá Colombia. 1998.

Programas en la Enseñanza de la Artes Plásticas-Ministerio de Educación Pública. 2001.

Klingler, Cynthia y Vadillo Guadalupe. Psicología Cognitiva. Estrategias en la práctica docente. México. Editorial Mc. Graw Hill. 1999. 